\title{
Partear y cuidar en Buenos Aires (1877-1920). Una aproximación comparativa
}

\section{Martin, Ana Laura}

Instituto Interdisciplinario de Estudios de Género

Facultad de Filosofía y Letras, Universidad de Buenos Aires, Argentina

lamartin1972@gmail.com

Cita sugerida: Martin, A.L.(2018).Partear y cuidar en Buenos Aires (1877-1920). Una aproximación comparativa Anuario del Instituto de Historia Argentina, 18 (1), e061. https://doi.org/10.24215/2314-257Xe061

Recibido:20 Marzo 2018 - Aceptado: 02 Mayo 2018 - Publicado: 28 de junio de 2018

(c) (1) (2) Esta obra está bajo licencia Creative Commons Atribución-NoComercial-CompartirIgual 4.0 Internacional http://creativecommons.org/licenses/by-nc-sa/4.0/deed.es_AR 


\title{
Partear y cuidar en Buenos Aires (1877-1920). Una aproximación comparativa
}

\section{Delivering babies and to take care in Buenos Aires (1877-1920). A comparative approximation.}

\author{
Ana Laura Martin \\ Instituto Interdisciplinario de Estudios de Género \\ Facultad de Filosofia y Letras, Universidad de Buenos Aires, Argentina \\ lamartin1972@gmail.com
}

\section{Resumen:}

Este artículo indagará sobre dos tareas realizadas casi exclusivamente por mujeres, la enfermería y la partería. Ambos oficios, entre el siglo XIX y XX, atravesaron un proceso de consolidación como tareas específicas en el esquema cada vez más jerarquizado de las profesiones médicas y vinculadas a la atención de la salud. En la ciudad de Buenos Aires el parto y el cuidado dejaron de ser actos individuales o de interés exclusivo de quienes necesitaban asistencia para convertirse en preocupaciones de otro orden, vinculadas con la higiene de la ciudad, la salud de la población y, en definitiva, el destino de la raza y de la Nación. De este modo, cuidar y partear, desarrollaron sus definiciones primeras que las encuadraron entre oficios calificados. En su versión moderna, ambas labores requerían algún tipo de capacitación y suponían un saber específico que las diferenciaba de las versiones previas, de las antiguas comadronas y de los improvisados cuidados inexpertos que ponían en peligro la vida de los niños, de las madres y de la población en general. Aquí se analizarán de manera comparativa los proyectos calificadores que involucraron a ambas tareas y los reposicionamientos que esto implicó para sus agentes. En el mismo sentido, se analizará el lugar que ocuparon los cuidados expertos y la atención de los partos en el sistema de atención de la ciudad de Buenos Aires.

Palabras clave: Enfermería, Siglos XIX-XX, Partería, Buenos Aires.

\section{Abstract:}

This article will explore two tasks performed almost exclusively by women, nursing and midwifery. Both trades, between the nineteenth and twentieth centuries, went through a process of consolidation as specific tasks in the increasingly hierarchical scheme of the medical professions and linked to health care. In the city of Buenos Aires, childbirth and care ceased to be individual acts or of exclusive interest to those who needed assistance to become concerns of another order, linked to the hygiene of the city, the health of the population and, ultimately, the destiny of the race and the Nation. In this way, caring for and starting, they developed their first definitions that framed them among skilled trades; in their modern version, both tasks required some type of training and implied a specific knowledge that differentiated them from the previous versions, from the old midwives and the improvised inexperienced care that endangered the lives of children, mothers and the population in general. Here we will analyze in a comparative way the qualifying projects that involved both tasks and the repositioning that this implied for its agents. In the same sense, the place occupied by expert care and delivery care in the care system of the city of Buenos Aires will be analyzed.

KeYwORDS: Nursery, Midwifery, Buenos Aires, 19th \& 20th Century.

\section{INTRODUCCIÓN}

\section{A fines de 1910 y frente a un auditorio de parteras, Cecilia Grierson afirmaba que:}

La profesión de partera, ha sido el primer oficio al que se han dedicado las mujeres y ha sido ejercida siglos de siglos, antes que sintiera la necesidad de la enfermera, que es una eflorescencia de la civilización moderna. Aquella, era llamada por una verdadera imposición de la naturaleza, ésta viene a ser un complemento en relación con nuestro progreso del último siglo. Una u otra, han ejercido su profesión, de una manera empírica, y ambas han tenido que ir poco a poco, fundando su arte sobre conocimientos científicos. (Grierson, 1903, p. 129).

Grierson señalaba los momentos de inflexión de dos ocupaciones por las que se interesó y con las que colaboró activamente: la partería y la enfermería. Desde la particular perspectiva de inicios de siglo en la 
Ciudad de Buenos Aires, la descripción expresa la diferencia entre dos profesiones ejercidas por mujeres y ubicadas en un escenario común, pero en posiciones distintas. Eso implicó recorridos que tuvieron semejanzas, aunque con alternativas y destinos diferentes para cada ocupación y para las mujeres incluidas en ellas.

Buenos Aires era una ciudad en plena modernización y su capacidad para atender las necesidades sanitarias de la población tenía límites. Hasta 1880 , la ciudad no podía garantizar la atención para la totalidad de las demandas ya que la infraestructura sanitaria y de elementos técnicos y profesionales no era suficiente (Gonzalez Leandri, Quirós y Suriano, 2010; Di Liscia, 2005). Cuando en 1892 Emilio Coni asumió su cargo como director en la Asistencia Pública registró 932 camas entre todos los hospitales de la ciudad, 72 de ellas destinadas a la atención de mujeres (Coni, 1918, p. 334). Pocos establecimientos porteños disponían de servicios de maternidad; el primero fue el Hospital de Mujeres, luego se crearon salas específicas en los Hospitales San Roque y Rawson que actuaban frente a los casos imperativos, pero debieron pasar varios años para que se dotara a Buenos Aires de un servicio de urgencias para partos. Fue el mismo Emilio Coni quien proyectó en 1892 la disposición de cuatro puestos fijos de parteras para atender las urgencias de la ciudad; en ese momento la población porteña alcanzaba 500.000 personas (Pérez, 1925, p. 7). Los cuidados en las instituciones no eran especializados; en muchos casos, estaba en manos de religiosas e incluso podían ser tareas ejercidas por mujeres encausadas o menores de edad a disposición de jueces correccionales (Wainerman, Geldstein, 1992). La enfermería fue una ocupación cuya calificación demoró varias décadas a pesar de la existencia de proyectos en esa dirección. La bibliografía sobre el tema en América Latina es reciente, pero permite señalar que la cuestión de la calificación y la definición de las tareas ha sido un asunto recurrente para la jerarquización de la enfermería como profesión (Eraso, 2013).

La delicadeza de asistir partos fue un asunto que resultó más urgente entre las consideraciones médicas de las autoridades sanitarias, de las instituciones públicas y de la beneficencia, que las actividades de cuidado en el hospital. Esto se tradujo en el interés por la educación de las parteras, la definición de sus funciones y la integración en el esquema de atención de la salud. Yolanda Eraso afirma que en grandes ciudades como Córdoba y Buenos Aires el problema de la infraestructura disponible no fue el único que capturó la atención de las autoridades, los recursos técnicos y profesionales fueron un asunto de interés entre las preocupaciones por la atención de las madres y los niños (Eraso, 2013). Se trata de tareas que es necesario pensar articuladas a fenómenos demográficos, sociales y políticos, que devienen en el desarrollo de las políticas públicas que relevan la preocupación por la higiene y la salud de los habitantes en el siglo XIX (Zamorano, 2011, p. 32).

Un arco variado de voces de médicos, políticos, mujeres vinculadas a la caridad, la filantropía y feministas, coincidieron en la preocupación por el modo en como los niños llegaban al mundo. Afirman Carolina Biernat y Karina Ramacciotti que la lucha contra la mortalidad materna e infantil, desde inicios del siglo XX, no sólo se circunscribió a la protección de las madres -sobre todo de las obreras, para evitar los efectos del trabajo femenino en la reproducción de la prole-, sino que también se extendió a un abanico de actores y problemas entre los cuales la asistencia de los embarazos, nacimientos y puerperios, tuvieron lugar como elemento de importancia dentro del sistema de atención de la salud. En este sentido, existe una vasta literatura que señala el interés creciente en el siglo XX por la salud de los niños y la multiplicación de la población (Biernat y Ramacciotti, 2013; Biernat y Ramacciotti, 2008; Armus y Belmartino, 2001; Álvarez, Molinari y Reinoso, 2004; Carbonetti, 2001; Becerra y Becerra, 2009). El trabajo de atender partos estuvo íntimamente ligado a ese nudo de problemas y así fue reconsiderado para devenir de modo definitivo en un asunto de la medicina y en un acto higiénico; esto significó definir los límites y las posibilidades del oficio de partear y el lugar de las mujeres en ellos (Nari, 2005; Eraso, 2013; Biernat y Ramacciotti, 2013). Como afirma María Soledad Zárate, se trató del pasaje de la "ciencia hembra a la ciencia obstétrica", de un saber y una actividad casi exclusiva de mujeres, con escasos límites y controles, en manos de un grupo heterogéneo de hacedoras, a un criterio definido, acreditado o "examinado" por la ciencia médica (Zárate Campos, 2007). 
El cambio de siglo abrió para ambas profesiones un nuevo escenario en el cual el Estado municipal, los médicos, y el sistema universitario y educativo tomaron un lugar definido -aunque no siempre efectivo- en la legitimación de sus funciones. La posibilidad de partear para las parteras dependió, cada vez más, de la legitimidad que le otorgara el reconocimiento de sus saberes y no sólo del prestigio que les proporcionaban sus antecedentes. La Ley del ejercicio de la medicina y sus ramas auxiliares, sancionada en 1877, fue un punto de partida para una serie de modificaciones en el modo de ejercer la profesión. Para las enfermeras la situación no fue la misma y los primeros años del siglo XX fueron decisorios para esta actividad. La posibilidad de dominar, "reivindicar y sostener, la exclusividad" de cuidar con ciertos niveles de autonomía resultó mucho más difícil -dilemático en términos de Eliot Freidson- para las enfermeras (Freidson, 1977). El análisis de este tipo de profesiones o proyectos profesionales en condiciones tan particulares, es decir en procesos de feminización y subordinación y dentro de esquemas jerárquicos como el de las ciencias médicas casi absolutamente ocupada por varones, merece un examen particular (Freidson, 1977, p. 79).

Aquí se propone una aproximación comparativa a las dos profesiones mencionadas por Cecilia Grierson, a través del análisis de la formación de enfermeras y parteras, en primer lugar, y del proceso de femeninización que implicó la formulación de ambas carreras durante la modernización del sistema de atención de la salud de la ciudad de Buenos Aires. Finalmente, se da cuenta de las estrategias que las agentes de ambas ocupaciones pusieron en juego a la hora de relacionarse con el sistema de atención y con las condiciones del ejercicio de la profesión. La dimensión comparativa persigue observar de manera particular asuntos generales, como puede ser la feminización de las tareas de cuidado en el ámbito de la atención de la salud, y también permite analizar las trayectorias específicas de la profesión. La información aportada por los censos nacionales y de la Ciudad de Buenos Aires, el discurso de médicos y funcionarios a través de artículos y tesis, y la producción de agentes de las profesiones aquí analizadas, son documentos que permiten avanzar en la dirección que el artículo propone.

\section{Ni empíricas ni universitarias}

La partería y la enfermería, comprendidas como profesiones calificadas y modernas, fueron incorporadas al sistema educativo formal cuando ambas ya existían y se habían desplegado en la ciudad. En ambos casos la instrucción formal fue producto de la intervención estatal en las formas de atender y administrar el dispositivo de atención de la salud.

Las parteras y comadronas fueron figuras asociadas a un hacer femenino antiguo, tradicional, compatible con prácticas domésticas y privadas. Así, el parto era un asunto femenino en manos de un universo variado de mujeres que habían adquirido experticia de diferentes modos. Algunas parteras estaban diplomadas, pero muchas estaban enroladas en lo que puede definirse como un variado grupo de personas dedicadas a partear sin ningún título o certificación habilitante. La tarea de estas mujeres, una vez iniciado el siglo XIX en la ciudad de Buenos Aires, fue paulatinamente asociada a lo "no científico", a lo "iletrado" y a la "ignorancia". Tanto diplomadas como no diplomadas fueron objeto de interés de la legislación porteña una vez disuelto el Protomedicato y organizado el Tribunal de Medicina en 1822 -con una importante influencia de los médicos porteños-, donde las reglamentaciones dispusieron la identificación y el registro de quienes ejercían el oficio de asistir partos y la obligatoriedad de tomar cursos especiales a todas las mujeres que quisieran hacerlo.

El Estado de Buenos Aires reconoció tempranamente la actividad y su expertise, la diferenció de otras profesiones como la de cirujanos y médicos, la subordinó al saber experto de los galenos e impuso obligaciones similares a las que corrían para otras tareas médicas. Según el Reglamento de Medicina de 1822, ejercer el oficio al margen de la ley podía ser considerado un acto criminal con pena de cárcel e inhabilitación. Estas medidas tempranas fueron de dificultosa aplicación, pero anticiparon lo que en el último tercio del siglo resultaría más palpable para las parteras: el control estatal y de la Facultad de Ciencias Médicas sobre sus tareas. En 1877 se consolidaron esas medidas con la Ley del ejercicio de la medicina y sus ramas auxiliares. 
Según la nueva ley, partear se definió como una rama menor dentro de la medicina, y por lo tanto sus intervenciones quedaron bajo la subordinación de otros saberes, en particular de los médicos. La normativa definió que para ejercer su profesión las parteras debían estar acreditadas por la Facultad de Ciencias Médicas de la Universidad de Buenos Aires, es decir, reforzó la práctica del registro y agregó restricciones concretas a la práctica de asistencia de partos. Las parteras sólo podían actuar en casos de partos normales, ya que frente a los laboriosos o distócicos estaban obligadas a dar parte a un médico. ${ }^{1}$

A las enfermeras esta situación no las alcanzó. El cuidado en instituciones o en el ámbito doméstico quedó fuera de la reglamentación. Sin embargo, a fines del siglo XIX, las instituciones dependientes de la Asistencia Pública reconocían el cuidado y la asistencia de enfermos como una actividad distinguible de otras que se desplegaban en sus dependencias. Con mayor o menor claridad, ejercido por diferentes actores y no siempre estrictamente ligado a la idea de "curar", el cuidado de enfermos era una labor demandada y practicada en los hospitales y asilos porteños. Esto explica el hecho de que la Asistencia Pública reconociera, a poco de su creación, que la dotación y formación de personal de enfermería era una cuestión pendiente a resolver. La frecuencia con la que se habilitaba a personas sin formación, "ignorantes y sin escuela", en las salas de los hospitales, señalaba las dificultades para establecer políticas sostenidas sobre el asunto (Penna y Madero, 1910, p. 143).

Quienes atendían los partos en la ciudad fueron parte de un mundo heterogéneo y, por lo tanto, el impacto de las normas y regulaciones fue desigual. Para algunas las nuevas condiciones -sobre todo el registro- fueron alcanzables y para otras significó ejercer su trabajo en condiciones marginales. Sin embargo, la matriculación y rematriculación exigida con mayor efectividad a partir de la década de 1880 expresó un sostenimiento y crecimiento de la actividad. El número de parteras matriculadas creció entre 1880 y 1890 de 294 a 466. 2 Por otro lado, el Censo General de Población, edificación e industrias de la ciudad de Buenos Aires, levantado en el año 1887, afirma que 164 mujeres declararon ser parteras en la Ciudad de Buenos Aires; este número triplicó el registrado por el Primer Censo Nacional de 1869. Estos datos, fragmentarios y provisorios, permiten afirmar que se mantuvo el interés por la profesión y que el circuito "credencializado" creció.

La Universidad tuvo un lugar clave en este proceso de ordenamiento ya que legitimó los saberes y organizó las relaciones entre médicos y parteras. La centralidad del sistema universitario en la consolidación de las profesiones modernas y su posibilidad de "estandarizar" el perfil de los expertos ha sido desarrollada por diferentes corrientes que analizan la cuestión. Sin embargo, sólo en algunas excepciones las tareas subordinadas y feminizadas como la de partear han sido estudiadas (Sarfatti Larson, 1977; Witz, 1992; Pozzio, 2016). La historiografía reciente en la región ha sido más prolífica en las últimas dos décadas y ha realizado algunos estudios de caso que son promisorios avances en la dirección del análisis sobre ocupaciones, donde la variable de género tiene un lugar relevante (Valobra y Ramacciotti, 2008; Valobra y Ramacciotti, 2010; Mott, 1999a; Mott, 1999b; Mott, 2001; Castro y Farias, 2009; Testa, 2012).

En el caso particular de Buenos Aires, recién a fines del siglo XIX los cursos de la Universidad se volvieron más estables, continuos y de obligatoriedad más efectiva, aunque hubo que esperar a la primera década del siglo XX para ver el funcionamiento pleno de una "verdadera escuela para partos" en la ciudad, dependiente de la Universidad de Buenos Aires y con maternidad anexa para atención y prácticas. Los datos de la Universidad, donde se dictaban los cursos que daban acceso a la matriculación, para este período son fragmentarios, y no es posible conocer con certeza el número de parteras que accedió a su diploma a través de esos cursos, pero si se toman como datos indíciales afirman la tendencia creciente. Durante la última década del siglo XIX el promedio de estudiantes de los "cursos de partos para señoras" fue de 41 por año, y en la primera década del siglo XX ascendió a $61 .^{3}$

Una hipótesis difícil de plantear, en términos cuantitativos, es que en la Ciudad de Buenos Aires eran menos las mujeres que estudiaban obstetricia que aquellas que obtenían su matrícula. Es decir, se mantuvieron las condiciones para que muchas mujeres lograran la habilitación para partear sin contar con un diploma, las medidas de regularización tendieron a homogeneizar lo que era heterogéneo, a ubicar en la misma condición 
a mujeres que tenían diferente formación y recorrido; o como describió un médico y autoridad universitaria, "desbrujar a las brujas" (Cantón, 1921, p. 562). Efectivamente existía esa posibilidad, y el Departamento Nacional de Higiene (DNH) mantuvo la prerrogativa de autorizar temporalmente a mujeres para que ejercieran el oficio, aunque no pudieran demostrar formación sistemática previa. Es decir, a pesar de que el sistema implementado para credencializar a las parteras tendía a que fuera la Universidad la principal agente de formación profesional, coexistieron otros modos de ingresar y mantenerse en el circuito legal de la atención del parto.

En el caso de las enfermeras el control y la capacitación no fueron un asunto de la Universidad. En este caso se trató una cuestión de la gestión municipal, un problema ligado a la administración hospitalaria, muchas veces en tensión entre la provisión de medios adecuados para la atención de la salud y la asignación de recursos materiales siempre escasos. En este sentido ocupó un lugar marginal si se compara con la situación de las parteras, quienes accedieron a cursos universitarios temprano.

La enfermería fue un asunto de menor preocupación para la agenda estatal y de la medicina. Sin embargo, la Asistencia Pública incorporó a su entramado la primera institución de la ciudad dedicada a formar enfermeras y enfermeros. Es un dato conocido el singular lugar que tuvo Cecilia Grierson como impulsora de la enfermería profesional y como alternativa laboral para sus congéneres. En efecto, la Escuela de Enfermeras, Enfermeros y Masajistas de la Ciudad de Buenos Aires, creada en 1885 pero incorporada a la órbita de la Asistencia Pública en 1892 por iniciativa de su titular Emilio Coni, la tuvo como principal mentora (Grierson, 1907; Morrone, 2013). Grierson había conocido el modelo inglés de enfermería, ligado fuertemente a la calificación, a la presencia femenina y a la atención hospitalaria.

Esa escuela fue por varias décadas la principal institución de capacitación de enfermeras. Estaba destinada a formar profesionales para los hospitales de la ciudad, aunque Grierson no descartaba que sus graduados y graduadas también pudieran ejercer como profesionales liberales para el público porteño. El Estado municipal reforzó la centralidad de la escuela cuando en 1905, a través de una ordenanza, exigió a las direcciones de los hospitales que enviran a sus enfermeros y enfermeras a cumplir los cursos de la escuela y diplomarse como condición para mantenerse en sus cargos.

El Estado porteño y Grierson coincidieron en la relevancia de calificar la profesión y ubicaron a la escuela como promotora de esa idea. Luego de la puesta en funcionamiento de la escuela de la Asistencia Pública otros establecimientos se organizaron con el propósito de calificar a sus propias enfermeras. A la escuela creada por Grierson se sumaron la Escuela de la Orden de la Conservación de la Fe (1914) dedicada a la formación de religiosas, un grupo de escuelas o de cursos regulares instalados en los hospitales como el Hospital Británico (1890), cursos en el Hospital Italiano (1903), y la Sociedad de Beneficencia (1916) que creó escuelas en sus diferentes dependencias. Más adelante, el Instituto de Medicina Experimental de la Universidad de Buenos (1923) instaló sus propios cursos de enfermería, exclusivos para ese centro de atención. La tendencia fue que cada hospital formara sus propias enfermeras y enfermeros.

\section{Los sentidos de la feminización}

Las interpretaciones que intentan explicar los motivos de la feminización de profesiones como la enfermería y partería ubican razones de orden muy diferente. Existen interpretaciones que afirman que es la fijación cultural y sexuada de roles de género lo que indicó capacidades "naturales" entre las mujeres para cuidar, y al mismo tiempo para ponerse al abrigo de órdenes superiores por su propensión a la "docilidad" y al altruismo (Mott, 1999a; Mott, 1999b; Mott, 2001; Mott, 2003). Otras sugieren que posiblemente hayan sido las necesidades de otros mercados de trabajo, que requerían mano de obra masculina, los principales impulsores de empleos femeninos como la enfermería (Castro y Farias, 2009). Otro tipo de estudios analizan las estrategias, más bien de orden colectivo, que implicaron un orden de género en este tipo de tareas y que aportan inteligibilidad a los comportamientos, en principio paradojales, de sus actoras. Esos estudios 
persiguen entender si fueron lógicas compensatorias las que impulsaron a las mujeres a inclinarse por oficios como los que aquí se analizan, como por ejemplo si entre las opciones que definieron las elecciones figuraron la posibilidad de ocupar posiciones en rangos menores de la jerarquía profesional a cambio de algún tipo de beneficio en el orden de las condiciones de trabajo (Witz, 1992; Pozzio, 2016). En este apartado se analizan los sentidos de la feminización para las ocupaciones tratadas, sin perder de vista los procesos específicos que atravesó cada una.

La asistencia del parto fue una experiencia femenina y así se mantuvo durante una parte importante del siglo XX. En una ciudad como Buenos Aires, los partos ocurrían de manera diversa. Las mujeres más privilegiadas podían elegir como parir, con frecuencia era en su propio domicilio y con la asistencia de alguna partera prestigiosa; en cambio las más pobres tenían menos alternativas y dependían más de las redes familiares o comunitarias. Hasta que la ciudad no contó con un sistema de emergencias para partos en la década de 1920, las mujeres que sufrían alguna complicación o carecían de asistencia, podían parir en las maternidades de la ciudad que a fines del siglo XIX eran muy pocas y con limitada cantidad de camas. El Hospital de Mujeres tenía la sala más grande de la ciudad, inicialmente con 8 camas, luego se sumó una sala especial en el Hospital Rawson con una capacidad similar (Arini, 1879, p. 24; Araya, 1904, p. 75; Parodi, 1878 , p. 8). Otra manera de atravesar el parto era en las casas de partos o en el domicilio de la partera. Las casas de partos eran pequeños establecimientos dirigidos por parteras - con frecuencia el propio domicilio de la profesional- que ofrecían sus servicios y que en algunos casos contaban con pensionado. Parir de esa manera era más accesible que contratando a una partera y fue una modalidad a la que recurrieron mujeres de modesta condición. Sin embargo, la medicina obstétrica que se desarrolló como especialidad médica comenzó a reclamar un lugar en el escenario del parto en el último tercio del siglo XIX, esto significó que en poco tiempo el parto deviniera en una incumbencia médica, tratado por parteras, pero bajo el dominio cognitivo de los galenos. En otros términos, la feminización de la partería tuvo como contrapartida la masculinización de la obstetricia.

El médico no era una figura totalmente ajena al parto asistido, antes del establecimeinto de la Ley del ejercicio de la medicina y sus ramas auxiliares, su presencia estaba limitada a las situaciones que la partera consideraba complejas. El médico y la partera, a veces socios, podían ser una dupla colaborativa y de mutua dependencia. En general fue la partera quien recomendó la asistencia de un médico a la parturienta o a su familia, pero la reglamentación sobre la práctica del parto alteró esa relación. La distinción entre la atención de los partos fisiológico y distócico o complicado alteró la sociedad previa entre médicos y parteras, que pasó a tener un signo diferente. En adelante, la partera para cuidar su sostenimiento en la profesión debió asegurarse la confianza de un médico que pudiera auxiliarla, de modo contrario corría el riesgo de incurrir en una falta que podía sacarla de la esfera legal.

Por otro lado, algo que completó la diferencia entre varones y mujeres a la hora de partear fue la enseñanza por separado. La obstetricia para médicos fue un asunto demorado en la Universidad porteña, y hasta 1892 se mantuvo la enseñanza mixta. Los futuros médicos y las "señoras que se dedicaban al arte de los partos" compartían las clases. Esto fue motivo frecuente de discusiones entre los profesores de obstetricia que, en general, coincidieron en reclamar la diferenciación de la enseñanza con argumentos que señalaban que era innecesario incluir algunos conceptos complejos en las clases de las parteras u obstétricas, y sobre todo aludían a la preparación previa que tenían las mujeres. Algunos afirmaban que las aspirantes "malamente sabían leer y escribir" y por lo tanto no podían "asimilar" del mismo modo que sus compañeros los conocimientos (Llames Massini, 1915, p. 169). La creación de la Escuela de Obstetricia en 1897, bajo la órbita de la Facultad de Ciencias Médicas de la Universidad de Buenos Aires, resolvió esta cuestión de manera definitiva diferenciando la enseñanza entre varones y mujeres, entre médicos y parteras u obstétricas. Las clases estaban completamente separadas y eran impartidas por cátedras diferentes, aunque durante los primeros años era usual que las parteras tomaran sus clases muy cerca de los futuros médicos, y por ello posiblemente el contacto e intercambio cotidiano se mantuvo frecuente. La maternidad anexa a la escuela fue la primera expresión 
concreta de la división del trabajo entre médicos y parteras, y también lo fue de la organización general y segmentada en términos de jerarquías y saberes en las instituciones especializadas. De la partera se esperaba que frente al parto normal se mantuviera una actitud expectante y paciente; en los casos más complejos, se pretendía que la partera profesional fuera la persona capaz de identificar la dificultad en un parto, aunque no atenderlo; la formación debía otorgarle capacidad de reacción frente a la urgencia y anticipación frente a la complicación. Por otro lado, la partera de la escuela era el principal nexo entre la parturienta y el médico, incluso dentro del hospital; era quien más horas pasaría en la sala o eventualmente en el hogar de la futura madre; de su capacidad de observación, de sus conocimientos y su experiencia dependía la intervención temprana del médico en una situación de riesgo. Como resultará claro más adelante, sobre las parteras se proyectó un lugar particular como ayudantes de los obstetras, complemento de su actuación en el parto. No sólo como propaladoras de saberes y criterios médicos establecidos y consensuados, se pretendía que pudieran actuar como vigilantes expertas del proceso del parto, como los ojos del obstetra en su ausencia. Este tipo de diferenciación en la formación se tradujo en las décadas siguientes en espacios de actuación segmentados para unos y otras, sobre todo en los ámbitos institucionales donde las parteras vieron cada vez más ajustadas sus funciones a las indicadas por el jefe de sala, y muchas veces limitadas incluso frente a prácticas menores. El obstetra, en cambio, se ubicó en el manejo cognitivo del parto, capaz de indicar y definir intervenciones y prácticas.

La feminización de la enfermería fue el resultado de un proceso donde las disposiciones del Estado porteño tuvieron un rol relevante al definir la profesión como exclusiva de mujeres en 1912. En adelante las instituciones de formación sólo se dedicaron a diplomar enfermeras. La presencia femenina en la ocupación venía en crecimiento en la ciudad: según la información censal en 1895 el 46\% de la enfermería estaba ocupada por mujeres, y en 1914 ese porcentaje ascendió al 58\%. Los enfermeros varones se mantuvieron en Hospital de Hombres y en algunos servicios específicos. Es decir, la intervención estatal colaboró a fijar una tendencia, la feminización de la profesión. Un factor de importancia para la decisión estatal fue la noción que se estableció sobre la ocupación, impulsada por Grierson a fines del siglo XIX, que logró mantenerse como referencia a inicios del siglo XX y que estaba asociada a la presencia de mujeres instruidas y al ejercicio en el hospital. El estado porteño ubicaba en sus instituciones de asistencia y atención las principales demandas y deficiencias del personal dedicado al cuidado, y apeló a las nociones que tenía disponibles para resolver la situación. La feminización de la educación de la enfermería dispuesta en 1912, cuyas medidas concretas se implementaron progresivamente, se produjo dentro de una renovación del impulso por dinamizar la profesión, calificarla y al mismo tiempo aumentar el número de sus agentes, siempre dentro del paradigma de la enfermería hospitalaria. Esto tuvo un impacto inicial favorable para los objetivos del Estado porteño, que vio crecer su matrícula. (Gráfico n. ${ }^{\circ} 1$ ).

Para ambos proyectos profesionales la feminización vino asociada a la calificación, significó mayores niveles de formación y acceso a posiciones de mayor prestigio. La aspiración moderna de ingreso al esquema de las profesiones sanitarias significaba que las enfermeras dejarían de ser asociadas a mucamas o simples sirvientas de sala, y que la partería quedaría asociada a una labor científica y de rigor médico monitoreada por profesionales. Sin embargo, esto tuvo sentidos encontrados para cada una de las tareas que aquí se analizan. Para la enfermería, abrió la posibilidad de una clara jerarquización respecto del punto de partida del oficio, y significó en los hospitales porteños el desplazamiento de los varones que hacían tareas similares y eran su principal competencia profesional. En el caso de las parteras, la dirección fue otra. El ingreso a las aulas universitarias y la creación de una escuela sólo para mujeres no significó la exclusividad sobre la atención de los partos sino que, por el contrario, fue sinónimo de ingreso de los obstetras al oficio. El ingreso a las aulas universitarias resultó en condiciones no del todo favorables, pues implicó compartir un campo con otros profesionales de mayor jerarquía y con quienes podían tener que disputar una clientela.

Para el caso porteño, las condiciones de trabajo y el modo en que enfermeras y parteras se vincularon con el sistema de atención terminaron de definir la orientación y el sentido que estas ocupaciones tomaron durante 
gran parte del siglo XX; y, en definitiva, las estrategias, decisiones individuales y grupales que las mujeres fueron capaces de desarrollar.

\section{Feminización, estrategias diferenciales y vinculación con el sistema de atención}

La información censal registra ambas tareas, enfermería y partería, y su análisis permite afirmar que las dos profesiones crecieron de manera sostenida, siendo mujeres de origen extranjero las más interesadas en ejercerlas. Las extranjeras aventajaron a las nacionales durante todo el período analizado, y la feminización se acentuó, aunque este es un proceso que debe individualizarse porque es necesario hacer lecturas diferentes para cada caso (Gráfico n. ${ }^{\circ} 2$ ).

Entre las enfermeras el incremento de la presencia femenina estuvo impulsada por el Estado porteño. Los datos que aquí se analizan toman sólo el inicio de ese proceso, a partir de 1912, y por lo tanto la presencia masculina aún fue alta (Gráfico n. $\left.{ }^{\circ} 1\right)$. En cambio, para el caso de las parteras lo que puede observarse a través de la información censal es el resultado del proceso de credencialización de la partería.

Esta tendencia al crecimiento de la obstetricia como opción profesional de mujeres, producida en un contexto de redefinición de las tareas obstétricas y de modificaciones del sistema santitario -en particular del aparato de cobertura de la madre y el niño-, reforzó la segmentación del trabajo obstétrico. Una división vertical entre las tareas de los médicos y parteras, marcada por la diferencia entre la eutocia y la distocia, y por la enseñanza separada, puso la actividad de las obstétricas bajo el dominio cognitivo del médico. Sin embargo, esa situación en principio no puso en peligro las posibilidades reales y materiales de las obstétricas. Los médicos no fueron una competencia preocupante para las diplomadas que tenían una clientela asegurada hasta los primeros años del siglo XX, y la mayoría de los partos porteños eran atendidos fuera de los hospitales donde las parteras tenían más chances de intervenir. El parto domiciliario se mantuvo como la forma más usual de parir hasta avanzado el siglo. Con esto claro, las parteras pretendieron mantener para sí a las personas que no recurrían al hospital como alternativa para atender los nacimientos. El parto "público" o "por la libre" fue el que aspiraron a dominar las obstétricas. Ofrecer sus servicios al público porteño como cualquier otra profesión liberal fue la estrategia de las parteras agremiadas en la Asociación Obstétrica Nacional (AON), la primera organización asociativa de parteras. ${ }^{4}$

La AON tuvo claro que la demanda estatal de parteras, principal empleador institucional, era limitada. Solo como ejemplo: la maternidad del Hospital Fernández, dirigida por Juan C. Llames Massini, contrató una sola partera en sus comienzos durante los primeros años de la década de 1920, y con el rango de segunda partera, pues las autoridades de la ciudad consideraban que su movimiento no ameritaba una partera jefa ni una primera partera (Llames Massini, 1930). La maternidad anexa a la escuela universitaria contrataba entre dos y cuatro parteras por turno con internado y contaba además con el sistema de rotación de estudiantes de obstetricia, que aliviaba las tareas de las titulares. Las instituciones de la Sociedad de Beneficencia estaban en una situación similar. En el censo porteño de 1904, 378 mujeres declararon ser parteras, resultando así evidente que el parto "por la libre" se mantenía como la principal opción de trabajo para las obstétricas, situación que se sostuvo por varios años. En 1926, cuando la ciudad tenía un sistema de atención maternal más desarrollado, con atención de urgencias a domicilio y una capacidad mayor a 500 camas, el municipio contabilizaba 55 parteras entre sus filas, aunque a la mayoría no le pagaba salario y la retribución era per cápita, es decir por cada parto atendido, lo que significaban $\$ 16$ por parto. La partera asalariada con mayor salario de la ciudad cobraba $\$ 250$, muy por debajo de lo que cobraban los médicos (entre $\$ 800$ y $\$ 600$ ) y por encima de lo que cobraban las enfermeras mejor pagas (\$180) (Ciudad de Buenos Aires, 1928).

Lo anterior explica que la $\mathrm{AON}$ tuviera entre sus preocupaciones, más que disputar el campo del parto con los obstetras, establecer la diferencia entre las diplomadas y las no diplomadas, perseguir el curanderismo obstétrico y asegurar condiciones de trabajo libres para sus socias. La estrategia fue cerrar las filas de la 
profesión y evitar el ingreso a sus filas de mujeres de menor calificación, insistir frente al DNH la aplicación estricta de las normas y no resignar el trabajo libre de partear en la ciudad. Así, en 1905 la AON propuso una reglamentación para el gremio que colocó a la autoridad del DNH como principal organismo de control y de admisión de parteras, definió las prácticas que concernían al ejercicio de la profesión y delineó algunos de los elementos de la ética profesional (Asociación Obstétrica Nacional, 1905, p. 110).

Según el proyecto de reglamentación, recaía en el DNH la responsabilidad de sancionar las normas que regularan el ejercicio de la partería y de hacerlas cumplir en las mismas condiciones que el resto de las profesiones médicas. Sería de incumbencia del Estado elaborar una lista de las parteras habilitadas y perseguir y sancionar los casos de incumplimiento de la ley. Respecto de la relación con los galenos y de las incumbencias de las parteras hubo una inevitable cuota de ambigüedad. En el primer artículo se consignaba que la partera estaría obligada a requerir la presencia médica siempre que el parto se complicara o que pudiera advertirse la peligrosidad de este. Reconocían con esto la necesidad de la presencia médica en casos de riesgo, pero convenían que el parto normal o eutócico era su terreno propio y exclusivo; en las más auspiciosas proyecciones, pretendían que sólo ellas fueran las ejecutoras de ese tipo de nacimientos. En este punto no disputaban con los médicos el tratamiento del partodistócico, pues conocían el riesgo que esto constituía para su condición, ya que era posiblemente el ámbito de mayor vulnerabilidad para las parteras debido a la enorme dificultad que significaba anticipar el riesgo en parto. Sin embargo, los partos normales podían ser de su absoluto dominio y en ellos se replegaron.

Otras voces ligadas al campo de la medicina y del feminismo local adoptaron una perspectiva diferente. Para Julieta Lanteri, la partería peligraba pues esa expertise se planteaba como prescindible frente al desarrollo de la medicina obstétrica. Advertía que la segmentación del trabajo del parto destinaba a las parteras los aspectos menos calificados de la tarea. El tiempo se convertía en el principal activo que las parteras tendrían para ofrecer, frente a la presencia del médico en el parto. La propuesta de Lanteri, propia de las feministas de su época, era que las mujeres se inclinaran por la medicina, que aspiraran a mayor calificación para no perder el dominio de la profesión (Lanteri, 1907).

Las enfermeras estuvieron frente a un escenario diferente. Su principal y casi único empleador fue el Estado municipal, con excepción de las instituciones de la Sociedad de Beneficencia y los hospitales de comunidad como el Italiano, Británico y Alemán que, en todos los casos, capacitaban a sus propias enfermeras. Para esta profesión, la regulación recayó más sobre las instituciones de formación y las direcciones institucionales que sobre las trabajadoras. Fueron las escuelas las que debieron garantizar la regularidad de sus cursos y quienes debieron validar sus diplomas ante el Estado. En general, esto se hizo a través de la Universidad de Buenos Aires con una modalidad diferente a la utilizada con las parteras. Un ejemplo es la escuela interna que funcionó en la Sociedad de Beneficencia (SB) dirigida por Lola Úbeda. Esa institución primero se ocupó de formar a las religiosas que oficiaban de enfermeras y, luego, en 1916, se estableció como Escuela Técnica de Enfermeras y el DNH, a través de la Universidad, oficializó sus títulos al año siguiente. La modalidad fue instalar un tribunal de evaluación al final de cada ciclo de formación que se ocupó de comprobar la suficiencia de cada graduada. Con esta modalidad se extendieron dentro de las instituciones de la SB varias escuelas de este tipo, aunque tuvieron serios problemas para su desarrollo y las mujeres convocadas no siempre respondieron positivamente a la instrucción propuesta. Al año siguiente de ser oficializada la Escuela Técnica de la SB, sus cursos no se pudieron dictar y las autoridades adujeron que fue porque las candidatas, enfermeras en ejercicio en la SB, se resistieron a matricularse. Las empleadas que ejercían cuidados en la SB reclamaron, en más de una oportunidad, por los bajos salarios que percibían, entre $\$ 40$ y $\$ 50$. Además, en tanto la instrucción no significara mejoras directas y concretas, era muy poco atractiva para las enfermeras en ejercicio. ${ }^{5}$

El modo de llegar a un puesto en las salas de los hospitales de la SB era como "ayudantes" sin salario, pero con la garantía de casa y comida en el lugar donde desarrollaban su tarea. Muchas veces eran las huérfanas que habían estado asiladas en la propia SB quienes, una vez mayores de edad, buscaban un modo de subsistir. Más adelante la práctica se afianzó y la SB recibió pedidos de los tribunales de menores para incorporar a 
jóvenes menores de edad recientemente huérfanas y sin familia, y solicitudes directas de mujeres jóvenes que buscaban un sustento y veían en la enfermería un modo de obtenerlo.

La práctica de la recomendación y/o madrinazgo tuvo un correlato en las condiciones de trabajo y en los conflictos gremiales motivados por las desigualdades que se generaron entre trabajadoras de condiciones muy diferentes. Si bien las enfermeras, en general, no promovían conflictos de este tipo, la SB enfrentó algunos ligados al escaso reconocimiento sobre las diplomadas y a las condiciones en las que ejercían su tarea. Las enfermeras que contaban con puestos fijos encontraban en sus compañeras de sala no diplomadas, como podían ser las "ayudantes" y las aprendices, una competencia real en el mercado laboral a la hora de exigir mejoras en sus condiciones de trabajo. En general, las aprendices y las no diplomadas o suplentes aceptaban largas jornadas de trabajo y guardias de más de 24 horas, además de bajos salarios. Todo esto significaba para las enfermeras titulares un obstáculo concreto para exigir mejores salarios, poniéndolas en una situación vulnerable frente a cada demanda. ${ }^{6}$

La propia Sociedad de Beneficencia, en reformas posteriores que planificó para sus Escuelas Técnicas, elaboró alternativas que no siempre logró poner en marcha. En la década de 1920, por ejemplo, proyectó un "Hogar de enfermeras" para uso de las enfermeras de la institución. ${ }^{7}$ Quienes se diplomaran tendrían luego de su graduación acceso al Hogar como complemento de sus tareas en el Hospital Rivadavia. Ese Hogar funcionaría como un servicio para el público y su recaudación sería compartida entre las enfermeras y la institución. Se trataba de una modalidad tendiente a mejorar las condiciones materiales de las enfermeras, evidentemente precarias.

En el caso de las instituciones de gestión estatal, la situación frente a la presión por la calificación se desarrolló de otro modo. Allí, desde la década de 1910, la Asistencia Pública exigía, con diferentes niveles de presión, que las trabajadoras pasaran por las aulas de la escuela municipal para poder seguir ejerciendo su tarea. El tratamiento errático sobre el personal de enfermería complicaba la administración institucional y tendía a abrir brechas entre lo dispuesto por las reglas y las necesidades de los hospitales, que en muchos casos no tenían más alternativa que relajar los criterios y las exigencias y mantener la convivencia entre diplomadas y no diplomadas. La tendencia de la administración municipal para que las trabajadoras de las instituciones de la ciudad se diplomaran y la enfermería adquiriera calificación encontró límites muy próximos. En el registro de egresos de la escuela municipal se puede ver con claridad el efecto normalizador y su proyección en los primeros años posteriores a 1916, cuando las medidas de feminización de la matrícula tuvieron su expansión completa, y cómo en poco tiempo declinó el número de graduadas. Cada vez menos mujeres se mostraron interesadas por obtener una certificación en enfermería, a pesar de que la escuela municipal había relajado sus niveles de exigencia y facilitado el ingreso y la permanencia (Gráfico n. ${ }^{\circ} 1$ ).

Las trabajadoras no se vieron seducidas por las nuevas condiciones, y las direcciones técnicas de los hospitales, más allá de estar de acuerdo con el criterio del gobierno porteño, no podían exigir que se cumplieran sus intenciones, pues necesitaban personal en las salas con o sin diploma. En definitiva, en las instituciones públicas y semipúblicas, coexistían diversas situaciones y condiciones que en su conjunto conspiraban contra la calificación profesional, o al menos la hicieron muy poco atractiva y dieron lugar a una dinámica que resultó difícil de romper. Era posible ocupar un puesto como enfermeras con escasos niveles de calificación, por lo tanto la instrucción se volvía muy poco interesante para las jóvenes. Por su parte, las instituciones necesitaron cada vez más este tipo de empleadas, y por eso recurrieron a incorporar en sus salas a mujeres con escasa instrucción, sorteando las dificultades normativas con distintos tipos de arreglos contractuales. 


\section{Cierre provisorio}

La tensión entre el impulso por calificar una tarea delicada como la de cuidar enfermos era cada vez más requerida en las instituciones porteñas, las posibilidades reales de dotar de ese personal formado a los hospitales y el interés de las mujeres convocadas a la enfermería imprimió una dinámica a la ocupación que resultó de largo plazo.

A pesar de los intentos del Estado porteño de calificar la tarea y desarrollar el perfil de la enfermera moderna o "profesional", y en definitiva crear la enfermería, la tendencia que se instaló fue otra, señalada por la capacidad de las instituciones de retener y, en el mejor de los casos, formar su propio personal. La escuela dependiente del municipio tuvo dificultades para resolver las necesidades de la ciudad y sus instituciones, y los hospitales de gestión no estatal mantuvieron la formación de su propio personal en las condiciones que consideraron más apropiadas y según sus necesidades. Esto estimuló muy poco el asentamiento de nuevos perfiles en enfermería alejados del hospital y dispuestos a atender necesidades y preocupaciones más amplias, relativas a la higiene urbana y de la población en general. La tendencia a la educación descentralizada y alejada de la Universidad no colaboró con la creación de modelos de enfermería consensuados para la ciudad capaces de atender lo que las autoridades sanitarias evaluaban necesario.

Enfermeras diplomadas, enfermeras de oficio, aprendices y personas sin mayor calificación convivieron en una tarea que no lograba definir incumbencias específicas ni convertirse en una verdadera preocupación para las autoridades sanitarias. El interés más nítido por definir la tarea provendría más adelante del interior de la actividad y de la elite de la profesión. La centralidad del sistema universitario para delinear el perfil profesional y definir incumbencias tuvo un rol destacado que diferencia el desarrollo de cada una de estas profesiones y que no jugó a favor de la enfermería.

Para las parteras las alternativas fueron otras. Durante las últimas décadas del siglo XIX, de modo paulatino la tarea libre de las parteras comenzó a experimentar límites que habían sido promovidos por dos instancias que coincidieron en su interés por los nacimientos: la medicina y sus instituciones, y el aparato estatal vinculado al control sanitario. El desarrollo de la obstetricia, como una especialidad de la medicina, llegó a la Universidad y a los hospitales, y con esto el parto dejó de ser un asunto sólo de mujeres. El ingreso de la obstetricia de los médicos varones llegó para instalarse en la escena del parto. Sin embargo, eso no significó el retiro de quienes siguieron siendo las ejecutoras de la atención del parto y las más cercanas a las mujeres durante ese trance. Con excepción del parto distócico, la atención se mantuvo en manos de mujeres y la tarea continuó siendo atractiva para ellas, que se ajustaron a las nuevas reglas. Posiblemente en la actividad cotidiana y concreta la función de partear haya cambiado poco; la mayoría de los nacimientos continuaron ocurriendo fuera de las instituciones, los procedimientos utilizados en los partos siguieron siendo limitados a una técnica y tecnología básica; los partos en su mayoría fueron normales, y la presencia médica obligada se mantuvo como excepción.

Las nuevas reglas y pautas modificaron la posición de las parteras frente al parto y a sus posibles socios, los médicos. En esta primera etapa definida por las regulaciones estatales las parteras no reaccionaron de modo colectivo, como lo hicieron algunos años después a través de las AON. Sin embargo, las estrategias individuales como matricularse y tomar los cursos que la Universidad ofrecía, adelantaron la dirección que las obstétricas tomarían. Custodiaron el ejercicio de la profesión en nuevos términos y se sumaron a las regulaciones participando ellas mismas, luego, de las demandas por la puesta en práctica de normas claras que las diferenciara de las "falsas parteras", y que al mismo tiempo les permitiera dominar un campo específico dentro de las tareas sanitarias. Un asunto en este punto, y que distingue las estrategias -o la falta de ellasde las enfermeras, es la capacidad que tuvieron las parteras de mantener su clientela y satisfacer esa demanda. Esto tiene origen en dos cuestiones: por un lado, en la capacidad técnica de atender satisfactoriamente los partos en términos de los conocimientos vigentes; por otro lado, en la falta de competencia concreta de los 
obstetras que no siempre estaban dispuesto a tolerar todas las instancias que la atención de una mujer en proceso de alumbramiento exigía.

En definitiva, las parteras entendieron que tenían un mercado disponible y, como he demostrado en otra parte, una razonable demanda por sus servicios hasta avanzados los años 20. Los obstetras y las instituciones estales tendrían que ofrecer mejores condiciones de atención, mayor seguridad en los partos y menos sufrimiento para que las mujeres los eligieran. Estas cuestiones se dirimieron en las décadas siguientes.

\title{
Gráficos
}

\author{
Gráfico n. ${ }^{\circ} 1$.
}

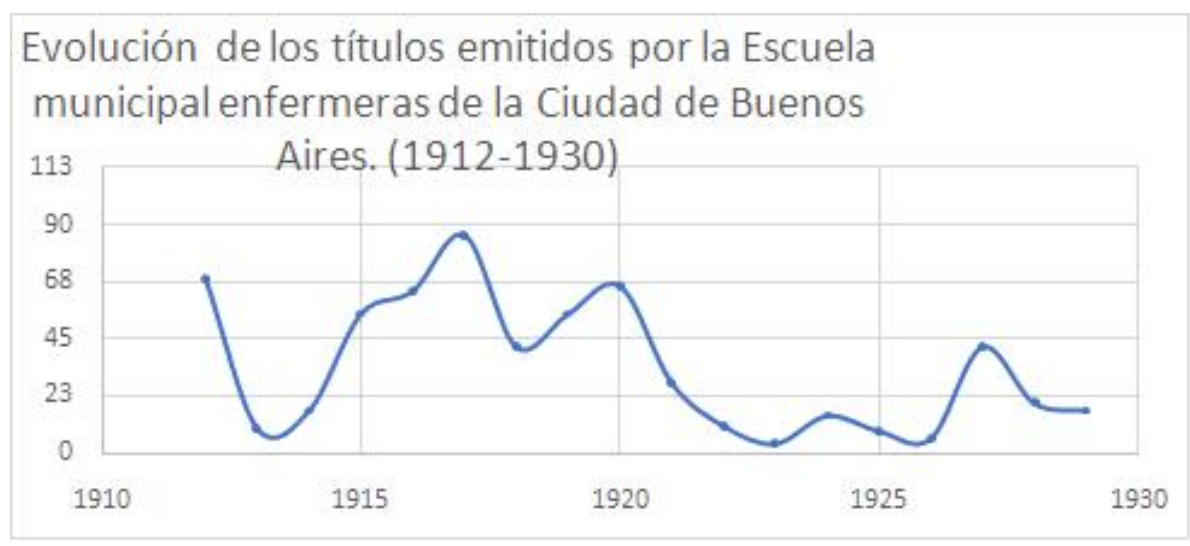

Elaboración propia.

Fuente: Libro de graduadas de la Escuela Municipal de Enfermeras “Cecilia Grierson”.

Gráfico n. ${ }^{\circ} 2$.

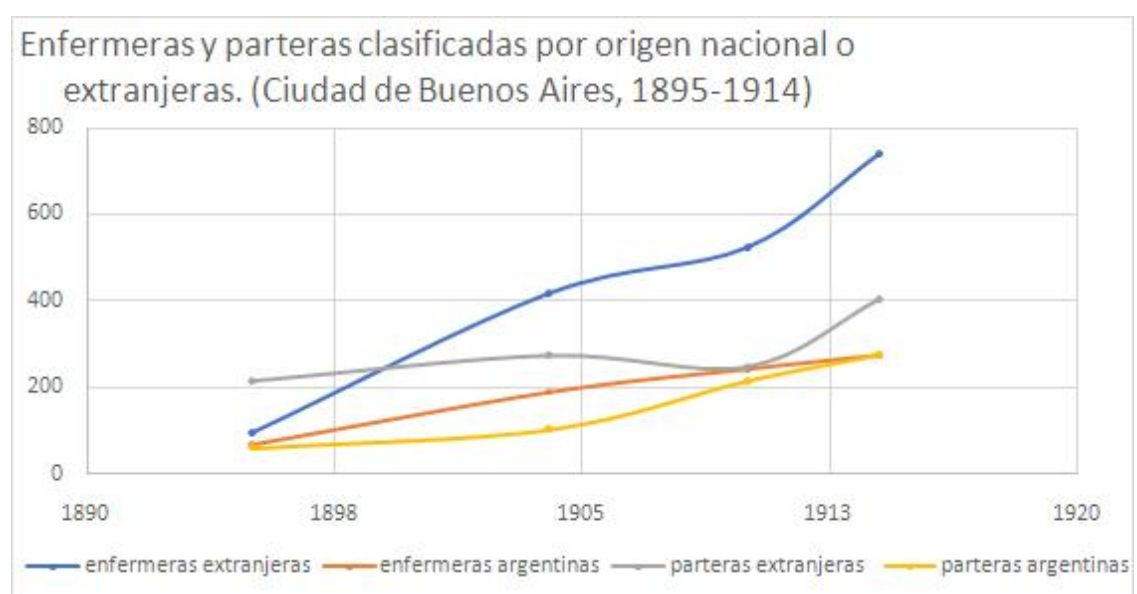

Elaboración propia.

República Argentina (1898). Segundo Censo de la República Argentina, Mayo de 1895. Buenos Aires, Taller tipográfico de la penitenciaria nacional, Tomo II.

Ciudad de Buenos Aires (1906). Censo General de Población, edificación, comercio e industrias de la Ciudad de Buenos Aires. Septiembre de 1904, Buenos Aires, Compañía sudamericana de billetes, Tomo segundo.

Ciudad de Buenos Aires (1910). Censo General de Población, edificación, comercio e industrias de la Ciudad de Buenos Aires. Octubre de 1909, Buenos Aires, Compañía sudamericana de billetes, Tomo segundo.

República Argentina, Comisión Nacional (1916). Tercer Censo Nacional de Población de la República. $1^{\circ}$ de junio de 1914, Buenos Aires, Talleres Gráficos Ross, Tomo IV. 


\section{REFERENCIAS}

Argentina, Departamento Nacional de Higiene (1881). Nómina de los médicos, farmacéuticos, parteras, dentistas y flebotomistas recibidos en la Facultad de Ciencias Médicas de Buenos Aires y de los veterinarios cuyos diplomas han sido registrados en 1881., Buenos Aires: Pablo E. Coni, 1881.

Argentina, Consejo de Higiene Pública. (1816), Nómina de los médicos, farmacéuticos, parteras, dentistas y flebotomistas recibidos en la Facultad de Ciencias Médicas de Buenos Aires y de los veterinarios cuyos diplomas han sido registrados en 1886., Buenos Aires: Europeaa, 1886.

Argentina, Departamento Nacional de Higiene (1890)., Nómina oficial de los médicos, farmacéuticos, parteras, dentistas y flebotomistas recibidos en la Facultad de Ciencias Médicas de la Nación y de los veterinarios cuyos diplomas han sido registrados y ley que reglamenta el ejercicio de estas profesiones en 1890., Buenos Aires, La Universidad, 1890.

Álvarez, A, Molinari, I., y Reinoso, D. (2004). Historia de enfermedades, salud y medicina en la Argentina de los siglos XIX-XX. Mar del Plata, Universidad Nacional de Mar del Plata.

Araya, R. (1904). Consideraciones clinicas y estadisticas sobre la Maternidad del Hospital San Roque en el año 1903. Tesis inaugural. Buenos Aires: Las ciencias.

Arini, J, B. (1879). Estudio estadístico. Hospital General de Mujeres. Tesis. Buenos Aires: Biedma.

Arini, J. B. y Rawson, G. (1879). Estudio estadístico. Hospital de Mujeres. Tesis para optar por al grado de doctor en medicina. Buenos Aires: Biedma.

Armus, D. y Belmartino, S. (2001). Enfermedades, médicos y cultura higiénica. En Alejandro Cattaruzza, Crisis económica, avance del Estado e incertidumbre politica (1930-1943). Buenos Aires: Sudamericana.

Asociación Obstétrica Nacional (1905). "Contribución a la ley en proyecto sobre ejercicio de la medicina y anexos. Presentado al Departamento Nacional de Higiene por la Asociación Obstétrica Nacional”. Revista Obstétrica, Año III, Tomo III.

Becerra Solá, M. y Becerra, N. (2009). Intervención social en la Argentina de los años 30: la profesionalización de la Asistencia Social. Universidad del Atlántico, Historia caribe, 15, 130-157.

Beruti, J. (1916). Nuestro gremio de parteras. Reformas necesarias para su mejoramiento y dignificación. Semana Médica, 23 (1), 9-38.

Biernat, C. y Ramacciotti, K. (2013). Crecer y multiplicarse. La politica sanitaria materno-infantil. Argentina, 1900-1960. Buenos Aires: Biblos.

Biernat, C. y Ramacciotti, K. (2008). La tutela estatal de la madre y el niño en la Argentina: estructuras administrativas, legislación y cuadros técnicos (1936-1955). História, Ciências, Saúde-Manghuinos, 2(15), 331-351. Recuperado de https://dx.doi.org/10.1590/S0104-59702008000200006

Cantón, E. (1921). La Facultad de Medicina y sus escuelas (Tomo VI). Buenos Aires: Coni.

Coni, E. (1918). Memoria de un médico higienista. Buenos Aires: Asociación Médica Argentina.

Carbonetti, A. (2001). La mortalidad infantil en la ciudad de Córdoba. Entre principios y mediados del siglo XX. En Sextas Jornadas Argentina de Estudios de Población (pp. 123-136). Neuquén: AEPA.

Castro, L. A. y Farias, L. (2009). A cooperação internacional e a enfermagem de saúde pública no Rio de Janeiro e Sao Paulo. En L. A. Castro y L. Farias, Saúdeß História. Sao Paulo: Hucitec.

Di Liscia, M. S. (2005). Dentro y fuera del hogar. Mujeres, familia y medicalización. Argentina, 1870-1940. Signos Históricos, 13, 94-119. http://www.redalyc.org/articulo.oa?id=34401305 (ultimo acceso 1 de febrero de 2017)

Eraso, Y. (2013). Representing Argentinian Mothers: Medicine, Ideas and Culture in the Modern Era, 1900-1946. Amsterdam, New York: Rapodi.

Eraso, Y.(2001).Ni parteras, ni médicos: obstetras. Especialización médica y medicalización del parto en la primera mitad del siglo XX. Anuario de la Escuela de Historia (Córdoba Argentina), 1(1), 109-124.

Freidson, E. (1997). La Profesión Médica. Barcelona: Península. 
Gonzalez Leandri, R. (1999). Curar, persuadir, gobernar. La construcción histórica de laprofesión médica en Buenos Aires, 1852-1886. Madrid: Biblioteca de la Historia de las Américas, Consejo Superior de Investigaciones Científicas.

GonzalezLeandri, R. (1984). Caridad y filantropía en la ciudad de Buenos Aires durante la segunda mitad del siglo XIX. En Armus, D. (comp.), Sectores populares y vida urbana (pp. 251-268). Buenos Aires: Consejo Latinoamericano de Ciencias Sociales.

González Leandri, R., González Bernaldo de Quirós, P. y Suriano, J. (2010). La temprana cuestión social. La ciudad de Buenos Aires durante la segunda mitad del siglo XIX. Madrid: CSIC.

Grierson, C., (1903). La historia de las obstétricas. Revista de Obstetricia, 1(I), 9-30

Grierson, C., (1907). Escuela de enfermeras. Comunicación presentada al Tercer Congreso Médico Latinoamericano reunido en Montevideo del 17 al 24 de marzo de 1907. Buenos Aires: Agustín Etchepareborda.

Lanteri, J. (1907). Moral obstétrica. Revista Obstétrica, V(4).

Llames Massini, J. C. (1915). La partera de Buenos Aires. Buenos Aires: Faliban y Camillioni.

Lorenzo, M. F. (2015). "Quésepa coser, que sepa bordar, que sepa abrir la puerta para ir a la Universidad". Las académicas en la Universidad de Buenos Aires en la primera mitad del siglo XX. Buenos Aires: Eudeba.

Martin, A. L. (2015). Parir, cuidar, asistir. El trabajo de las parteras y enfermeras en Buenos Aires (1877-1955) (Tesis de doctorado). Facultad de Filosofía y Letras, UBA.

Martin, A. L. (2015). Mujeres y enfermería: una asociación temprana y estable (1886-1940). En C. Biernat, J. M. Cerdá y K. Ramacciotti (dir), La salud pública y la enfermería en la Argentina (pp. 257-285). Buenos Aires: UNQUI.

Morrone, B. (2013). Soltando amarras. Claves para comprender la historia pendiente de la enfermería. Buenos Aires: Ed. Suárez.

Mott, M. L. (1999). Revendo a história da enfermagen em Sao Paulo (1890-1920). Cadernos Pagú, 13, 327-345.

Mott, M. L. (1999). O curso de Partos: Debe ou nao haver parteiras? Cadernos de Pesquisam, 108, 133-160.

Mott, M. L. (2001). Fiscalizacao e Formação das parteiras em Sao Paulo (1880-1920). Revista de la Escola de Enfermagem, 1(35), 46-53.

Mott, M. L. (2003). Midwifery and the Construction of Image in Nineteenth-Century Brazil. Nursing History Review, 11, 31-49.

Nari, M. A. (2005). Las políticas de la maternidad y maternalismo político.Buenos Aires, 1890-1940. Buenos Aires: Biblos.

Parodi, A. (1878). Estudio estadístico sobre la maternidad del Hospital General de Mujeres. (Tesis de doctorado). Buenos Aires: Coni.

Penna, J. y Madero, H. (1910). La administración Sanitaria y Asistencia Pública de la Ciudad de Buenos Aires. Tomo II. Buenos Aires: Kraft.

Pérez, M. L. (1925). La asistencia maternal a domicilio en la Ciudad de Buenos Aires. Buenos Aires: Concejo Deliberante de la Ciudad de Buenos Aires.

Pozzio, M. (2016). Análisis de género y estudios sobre profesiones: propuestas y desafíos de un diálogo posible -y alentador- (en línea). En Mujeres, trabajo y profesionalización. Recuperado de http://historiapolitica.com/dossi ers/mujeres-y-profesionalizacion/ (último acceso: 26 de diciembre de 2016)

Ruggiero, K. (1992). Honor, Maternity, and the Disciplining of Women: Infanticide in Late Nineteenth-Century Buenos Aires. The Hispanic American Historical Review, 72(3), 353-373.

Sarfatti Larson, M. (1977). The Rise of Professionalism: A Sociological Analysis. California: Universityof California.

Testa, D. (2012). Aportes para el debate sobre los inicios de la profesionalización de la terapia ocupacional en Argentina. Revista Chilena de Terapia Ocupacional, 12(1), 72-87.

Universidad de Buenos Aires (1889). Anales de la Universidad de Buenos Aires. Tomo III, Buenos Aires,

Universidad de Buenos Aires (1891). Anales de la Universidad de Buenos Aires. Tomo VI, Buenos Aires: Biedma,

Universidad de Buenos Aires (1892). Anales de la Universidad de Buenos Aires. Tomo VII, Buenos Aires Universidad de Buenos Aires: Biedma, 
Universidad de Buenos Aires (1893). Anales de la Universidad de Buenos Aires, Tomo X, Buenos Aires: Biedma Universidad de Buenos Aires (1894). Anales de la Universidad de Buenos Aires, Tomo X, Buenos Aires: Biedma. Universidad de Buenos Aires (1896). Anales de la Universidad de Buenos Aires, Tomo XII, Buenos Aires: Biedma. Universidad de Buenos Aires (1897). Anales de la Universidad de Buenos Aires, Tomo XIII, Buenos Aires: Biedma. Universidad de Buenos Aires (1899). Anales de la Universidad de Buenos Aires, Tomo XIV, Buenos Aires: Biedma. Universidad de Buenos Aires(1890). Anales de la Universidad de Buenos Aires, Tomo XV. Buenos Aires: Biedma. Universidad de Buenos Aires (1902). Anales de la Universidad de Buenos Aires, Tomo II. Buenos Aires: Biedma. (Universidad de Buenos Aires 1914) Revista de la Universidad de Buenos Aires, Tomo XXVII. Buenos Aires: Biedma. Valobra, A. y Ramacciotti, K. (2010). La profesionalización de la enfermería en Argentina: disputas institucionales y políticas durante el peronismo. Asclepio, 62(2).

Valobra, A. y Ramacciotti, K. (2015). Feminización y profesionalización de la enfermería (1940-1955). En C. Biernat, J. M. Cerdá y K. Ramacciotti (dir), La salud pública y la enfermería en la Argentina (pp. 287-314). Buenos Aires: UNQUI.

Valobra, A. y Ramacciotti, K. (2008). Profesión, vocación y lealtad en la enfermería peronista. En C. Biernat, Ramacciotti K. y Valobra, A., La Fundación Eva Perón y las mujeres: entre la provocación y la inclusión (pp. 119-150). Buenos Aires: Biblos.

Wainerman, C. H., y Geldstein, R. N. (1990). Condiciones de vida y de trabajo de las enfermeras en la Argentina. Cuadernos del CENEP, 44.

Wainerman, C. H., y Geldstein, R. N. (1992). El nacimiento de una ocupación femenina: la enfermería de Buenos Aires. Desarrollo Económico, 32(126), 271-284.

Witz, A. (1992). Professions and Patriarchy. London: Routledge.

Zamorano, P. (Ed) (2011). Vencer la cárcel del seno materno. Nacimiento y vida en el Chile del Siglo XVIII. Santiago de Chile, Universidad de Chile.

Zárate Campos, M. S. (2007). Dar a luz en Chile, siglo XIX. De la "ciencia hembra" a la ciencia obstétrica. Santiago de Chile: Ediciones Universidad Alberto Hurtado.

\section{Notas}

1 La Asociación Obstétrica Nacional (AON) se creó en 1901 por parteras diplomadas. Fue una organización autónoma e independiente, pero que contó con el apoyo directo de los principales maestros de obstetricia, profesores de escuela, de la cátedra de Obstetricia de la Facultad de Medicina y de médicas como Julieta Lanteri y Cecilia Grierson, que fue su presidenta honoraria. La AON existe en la actualidad.

2 Archivo General de la Nación, Sociedad de Beneficencia, Legajo № 244.

3 Archivo General de la Nación, Sociedad de Beneficencia, Legajo No 162.

4 Archivo General de la Nación, Sociedad de Beneficencia, Legajo No 244.

5 Argentina, Ley de Reglamento del Ejercicio de la Medicina, Farmacia y Demás Ramos del Arte de Curar. Capítulo IV, 1877. La misma ley sufrió varias modificaciones, la más inmediata en 1912, sin que se alterasen los artículos relativos al arte de partear.

6 El cálculo es propio a partir de la Nómina de los médicos, farmacéuticos, parteras, dentistas y flebotomistas recibidos en la Facultad de Ciencias Médicas de Buenos Aires.

7 El cálculo es propio a partir de los datos de Anales de la Universidad de Buenos Aires y Revista de la Universidad de Buenos Aires. 